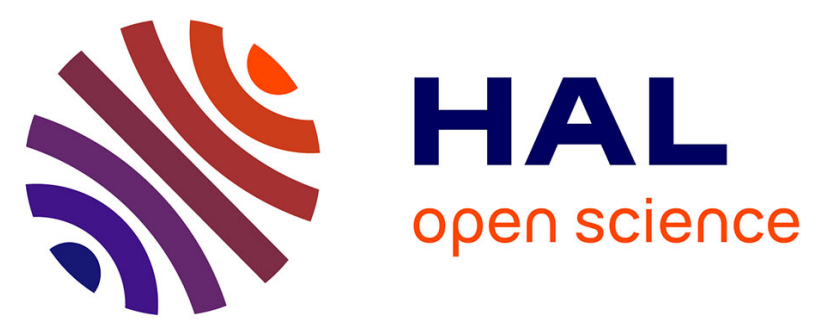

\title{
ANALYSE LEXICALE DE LA QUALITÉ DE VIE AU TRAVAIL DES PSYCHIATRES À L'HÔPITAL
}

Elisanne de Gantes, Gauthier Bellagamba, Marie-Pascale Lehucher-Michel

\section{To cite this version:}

Elisanne de Gantes, Gauthier Bellagamba, Marie-Pascale Lehucher-Michel. ANALYSE LEXICALE DE LA QUALITÉ DE VIE AU TRAVAIL DES PSYCHIATRES À L'HÔPITAL. Santé Publique, 2014, 26, pp.803-812. hal-01243052

\section{HAL Id: hal-01243052 \\ https://hal-amu.archives-ouvertes.fr/hal-01243052}

Submitted on 14 Dec 2015

HAL is a multi-disciplinary open access archive for the deposit and dissemination of scientific research documents, whether they are published or not. The documents may come from teaching and research institutions in France or abroad, or from public or private research centers.
L'archive ouverte pluridisciplinaire HAL, est destinée au dépôt et à la diffusion de documents scientifiques de niveau recherche, publiés ou non, émanant des établissements d'enseignement et de recherche français ou étrangers, des laboratoires publics ou privés. 
CHERCHER, REPÉRER, AVANCER

\section{ANALYSE LEXICALE DE LA QUALITÉ DE VIE AU TRAVAIL DES PSYCHIATRES À L'HÔPITAL}

Elisanne De Gantes, Gauthier Bellagamba, Marie-Pascale Lehucher-Michel

S.F.S.P. | « Santé Publique »

2014/6 Vol. 26 | pages 803 à 812

ISSN 0995-3914

Article disponible en ligne à l'adresse :

http://www.cairn.info/revue-sante-publique-2014-6-page-803.htm

\section{!Pour citer cet article :}

Elisanne De Gantes et al., «Analyse lexicale de la qualité de vie au travail des psychiatres à l'hôpital », Santé Publique 2014/6 (Vol. 26), p. 803-812.

Distribution électronique Cairn.info pour S.F.S.P..

(C) S.F.S.P.. Tous droits réservés pour tous pays.

La reproduction ou représentation de cet article, notamment par photocopie, n'est autorisée que dans les limites des conditions générales d'utilisation du site ou, le cas échéant, des conditions générales de la licence souscrite par votre établissement. Toute autre reproduction ou représentation, en tout ou partie, sous quelque forme et de quelque manière que ce soit, est interdite sauf accord préalable et écrit de l'éditeur, en dehors des cas prévus par la législation en vigueur en France. Il est précisé que son stockage dans une base de données est également interdit. 


\title{
Analyse lexicale de la qualité de vie au travail des psychiatres à I'hôpital
}

\section{Lexical analysis on the quality of work life of hospital psychiatrists}

\author{
Elisanne De Gantes ${ }^{1}$, Gauthier Bellagamba ${ }^{1,2}$, Marie-Pascale Lehucher-Michel ${ }^{1,2}$
}

\section{$\sqsubset \rightarrow$ Résumé}

Objectif : Une enquête qualitative a été réalisée en 2013 auprès de psychiatres hospitaliers travaillant dans deux établissements de régions différentes. L'objectif était de documenter la qualité de vie au travail des psychiatres des établissements publics hospitaliers.

Méthodes : Des entretiens semi-directifs ont été menés auprès de psychiatres à l'aide d'un guide d'entretien. Ces derniers ont été tirés au sort en tenant compte de l'établissement, de la tranche d'âge, du genre et de la spécialité adulte ou pédiatrique. Le contenu des données a été analysé à l'aide du logiciel Alceste puis interprété.

Résultats : Seize psychiatres ont été interrogés. Quatre thèmes, constitués de sous-thèmes, ont été mis en évidence. Le premier concerne la prise en charge médico-sociale du patient, avec l'environnement socio-familial de l'enfant, les contraintes structurelles et les interactions avec le réseau de soins. Le second est relatif à la qualité des soins avec la formation des professionnels de santé et la dimension relationnelle. L'exercice dans le système public hospitalier constitue le troisième thème avec le rôle du médecin-gestionnaire, la perte de sens du travail et la satisfaction au travail. Le dernier thème fait référence à la spécificité du travail en psychiatrie avec les aspects médico-légaux et le problème de la violence.

Conclusions: Cette enquête suggère que les psychiatres sont globalement satisfaits de leur activité professionnelle. Les éléments à améliorer seraient une organisation du temps de travail favorisant l'écoute des patients, les échanges interprofessionnels et la formation du personnel paramédical. Ces résultats sont à confirmer par une étude quantitative.

Mots-clés: Satisfaction professionnelle; Qualité de vie; Psychiatrie; Hôpitaux psychiatriques; Recherche qualitative.

\section{$\hookrightarrow$ Summary}

Purpose: A qualitative study was conducted in 2013 with hospital psychiatrists working in two psychiatric hospitals of different regions in order to document the quality of work life of public hospital psychiatrists.

Method: Semi-guided interviews were conducted with psychiatrists using an interview guide. Physicians were randomized according to health institution, age, gender and adult or paediatric psychiatry speciality. Data were analysed with the Alceste software and interpreted.

Results: Sixteen psychiatrists were interviewed. Four themes, composed of sub-themes, were highlighted. The first them concerned medical and social patient care, with the child's social and family environment, structural constraints and interactions with the care network. The second them concerned quality of care with the training of healthcare workers and the relational dimension. The third them concerned working in the public hospital system with the role of the "physician-administrator", loss of the meaning of work and job satisfaction. The last theme refers to the specificity of working in psychiatry with forensic aspects and violence issues.

Conclusion: This study suggests that psychiatrists are globally satisfied with their jobs. The main factors to be improved are working time organization to allow more time to listen to the patient, interprofessional exchanges and paramedical staff training. These data should be assessed by means of a quantitative study.

Keywords: Job satisfaction; Quality of life; Psychiatry; Hospitals, psychiatric; Qualitative research.

\footnotetext{
${ }^{1}$ AP-HM - Timone - Service de Médecine et Santé au travail - 264, rue Saint-Pierre - 13385 Marseille - France.

${ }^{2}$ Aix-Marseille Univ. - EA 3279 - Santé publique - Marseille - France. 
L a qualité de vie au travail est un concept intuitif et dynamique fondé sur la perception qu'a un individu de sa place dans les sphères culturelle, organisationnelle et relationnelle de l'entreprise [1]. Les risques psychosociaux entendus comme les risques engendrés par les conditions d'emploi, l'organisation et les relations au travail sont des déterminants délétères de la qualité de vie au travail [2]. En France, le concept de qualité de vie au travail a été introduit depuis quelques années dans les critères de certification des établissements de santé. En effet, la qualité de vie au travail des professionnels est nécessaire à une bonne qualité des soins et la délivrance de soins de qualité est nécessaire pour que les professionnels perçoivent une bonne qualité de vie au travail [3].

Selon deux enquêtes françaises réalisées en 1998 puis en 2003, la perception que le personnel hospitalier a de ses conditions de travail au cours de cette période, s'est globalement dégradée : rythmes plus contraignants, manque d'effectifs, etc. [4]. Concernant l'univers médical, de nombreux bouleversements sont apparus, en lien avec une augmentation des exigences et des contraintes diverses: responsabilités médicales, surcharge administrative, altération de la relation médecin-patient, pression politique et sociale, etc. Les médecins ont une faible capacité à s'avouer leur propre souffrance psychologique. Pourtant une étude française montre que chez les médecins, le taux de prévalence du syndrome d'épuisement professionnel est de $43 \%$, des troubles psychologiques de $29 \%$ et d'une médiocre santé perçue pour $8 \%$ [5].

Plus spécifiquement, la psychiatrie, au sein d'une profession médicale déjà à risque, semble particulièrement exposée aux risques psychosociaux [6]. Dans la littérature internationale, des études montrent que la psychiatrie est une spécialité à haut risque d'épuisement professionnel $[5,10]$ et près de $35 \%$ des psychiatres en état d'épuisement professionnel ne pensent pas l'être [6]. Elle constitue également une des spécialités médicales les plus à risque de suicide [11-13]. La littérature a mis en évidence des risques spécifiques pour la santé des médecins psychiatres [14-16] : exposition à la souffrance psychique et à la chronicité des troubles mentaux, charge émotionnelle intense de la relation médecin-patient, confrontation à la violence, au suicide et gestion du passage à l'acte. Ils doivent également faire face à la fois à la stigmatisation de la population qu'ils traitent et à celle de leur propre spécialité. Enfin, il existe un amalgame fréquent entre les pathologies psychiatriques et les problèmes d'ordre social ou judiciaire. Les facteurs de l'environnement de travail associés au syndrome d'épuisement professionnel diffèrent entre les psychiatres et les autres praticiens.
Pour exemple, chez les psychiatres, la durée de travail hebdomadaire entre 35 et 50 heures et un nombre de nuits travaillées de plus de cinq par mois apparaissent plus en lien avec ce syndrome alors que chez les autres spécialistes, la violence de la part des patients et la pression temporelle élevée apparaissent plus en lien avec ce dernier [5].

La plupart des études sur le vécu des conditions de travail en milieu hospitalier psychiatrique concerne les soignants paramédicaux $[17,18]$ et très peu les psychiatres.

L'objectif de notre étude est de documenter la qualité de vie au travail des psychiatres des établissements publics hospitaliers afin de fournir des éléments pour préparer une enquête quantitative.

\section{Méthodes}

\section{Type d'enquête}

Il s'agit d'une enquête qualitative réalisée à l'aide d'entretiens semi-directifs. Ce type d'entretiens permet le recensement de données subjectives et s'attache à recueillir les éléments mis en avant par la personne interrogée dans des thématiques relativement larges [19]. Une étude qualitative va permettre de générer des hypothèses conduisant dans un second temps à l'élaboration d'items qui seront intégrés à un questionnaire quantitatif [20]. Une fois finalisé, ce questionnaire pourra être appliqué à l'ensemble de la population étudiée afin de tester ces hypothèses.

\section{Population}

Un entretien a été proposé à des psychiatres de deux établissements publics de santé mentale du sud de la France. L'un des deux établissements, intégré dans un centre hospitalo-universitaire et intervenant en secteur urbain, est en déficit avec un plan de retour à l'équilibre. Les postes de psychiatres de cet hôpital sont pourvus. Le second établissement, non universitaire et intervenant en milieu rural, n'est pas en déficit et est également correctement pourvu en praticiens (un seul poste vacant lors de l'enquête). Les psychiatres ont été tirés au sort parmi l'ensemble des psychiatres travaillant dans ces structures depuis au moins six mois. Afin de constituer l'échantillon, compte tenu des ressources importantes nécessaires pour réaliser les entretiens semi-directifs notamment les temps d'entretien et de saisie et en vue de respecter le principe de faisabilité de l'étude, quatre facteurs de confusion ont 
été sélectionnés par le comité de pilotage : l'établissement, la spécialisation en psychiatrie adulte ou en pédopsychiatrie, le genre et la tranche d'âge $(<35$ ans ou $>=$ 35 ans). La prise en compte de ces facteurs (quatre facteurs à deux modalités chacun) a nécessité l'inclusion de 16 sujets. Seize groupes, comportant une liste de quatre psychiatres tirés au sort, ont été constitués. Un rendezvous a été proposé au premier psychiatre de la liste. En cas de refus ou de non réponse, le second psychiatre de la liste a été sollicité et ainsi de suite.

\section{Recueil des données}

Un guide d'entretien a été élaboré par deux spécialistes en médecine du travail, une psychologue et un épidémiologiste suivant trois étapes :

1) L'identification des thématiques à explorer à partir d'une analyse des facteurs de risque psychosociaux, identifiés par la Direction de l'animation de la recherche, des études et des statistiques, et à partir d'une revue de la littérature internationale sur les conditions d'exercice et le vécu des psychiatres hospitaliers.

2) L'analyse critique des thématiques par trois psychiatres des établissements étudiés.

3) L'application du guide afin de le tester auprès de deux psychiatres de ces établissements.

Le guide d'entretien aborde ainsi les huit thématiques suivantes : ressenti au travail et difficultés rencontrées dans l'exercice de la profession, impact de l'organisation du travail sur la façon de travailler, qualité des soins, relations interprofessionnelles, relations avec les patients et leur entourage, liens entre vie professionnelle et vie personnelle, influence du travail sur la santé, préconisations pour améliorer la qualité de vie au travail.

En avril et mai 2013, des entretiens semi-directifs confidentiels ont été conduits, pendant le temps de travail des médecins, par une interne en médecine du travail auprès de psychiatres tirés au sort ayant accepté de participer à l'étude. Ils ont été réalisés dans une salle réservée à cet effet dans les deux établissements. L'objectif de l'étude a été précisé dans une lettre d'information à chacun d'entre eux lors de la proposition d'entretien. Les entretiens ont été enregistrés avec leur accord et le principe de l'anonymat a été garanti.

\section{Analyse des données}

Le contenu des entretiens a été saisi informatiquement dans son intégralité par l'enquêteur. L'analyse de contenu des discours a été réalisée selon la méthode de l'analyse lexicale et thématique qui permet d'étudier la structure grammaticale de chaque entretien, la richesse du vocabulaire employé et de mettre en évidence les thématiques évoquées [21]. Cette analyse, effectuée à l'aide d'un logiciel Alceste, se décompose en quatre phases [22] :

- Élaboration de la liste des mots employés réduits à leur racine ;

- Découpage du texte en unités de contexte élémentaire (correspondant à des phrases extraites des entretiens) puis regroupement des unités contenant les mêmes formes lexicales afin de constituer des classes de mots dont le sens sera ensuite attribué par l'analyste ;

- Pour chaque classe de mots obtenue, classement des mots sur la base de leur représentativité déterminée par un test de Khi2 à partir du nombre d'occurrences et de co-occurrences (fréquence de survenue de mots associés ensemble) ;

- Extraction des unités de contexte élémentaire les plus représentatives de chaque classe en fonction de leur valeur de Khi2.

La valeur du Khi2 exprime le coefficient d'association entre une unité de contexte élémentaire et une classe. Par convention, la valeur minimum du Khi2 est déterminée lorsque $70 \%$ des unités de contexte élémentaire sont classées; chaque classe étant ainsi considérée comme stable [23]. Dans le cas de cette étude, la valeur minimum du Khi2 est de 16. Pour l'interprétation des données, seuls les mots et unités de contexte ayant une valeur de Khi2 supérieure à 16 ont donc été pris en compte. À partir des classes de mots obtenues, deux spécialistes en médecine du travail, une psychologue et un épidémiologiste ont structuré l'analyse en thèmes et sous-thèmes.

\section{Aspects éthiques}

La participation était volontaire et anonyme, conformément aux dispositions de la loi du 6 août 2004. Le traitement des données ayant servi à cette étude a été déclaré à la commission nationale de l'informatique et des libertés.

\section{Résultats}

Afin de pouvoir inclure 16 sujets dans l'étude, 24 psychiatres ont dû être contactés. Huit psychiatres de chaque établissement ont accepté de participer à l'enquête (taux de réponse : $67 \%$ ). Parmi les huit qui n'ont pas 
répondu favorablement à la proposition d'entretien, trois ont déclaré ne pas pouvoir se libérer durant les créneaux proposés, trois ont indiqué ne pas en avoir le temps et deux ont été injoignables. La durée des entretiens a varié de 20 à 90 minutes (moyenne et écart-type en minutes : $46,8+/-19,8)$.

Les caractéristiques socioprofessionnelles (sexe, âge, spécialisation, secteur d'activité principale, fonction) des médecins interrogés sont les suivantes : huit femmes, onze psychiatres âgés d'au moins 35 ans, onze travaillant en psychiatrie adulte, douze exerçant principalement en intrahospitalier et quatre en centre médico-psychologique (CMP). Parmi eux, onze sont praticiens hospitaliers, trois chefs de pôle, un chef de clinique-assistant et un professeur des universités - praticien hospitalier. Quatre thèmes ont été mis en évidence à la suite de l'interprétation des données fournies par Alceste. Chaque thème est constitué de sous-thèmes caractérisés par les occurrences et co-occurrences de mots les plus fréquentes selon les résultats de l'analyse du logiciel Alceste (tableau I).

Les thèmes 1 et 2 , qui représentent respectivement $22 \%$ et $19 \%$ du corpus, concernent principalement la pédopsychiatrie, avec la prise en charge médico-sociale du patient et la qualité des soins. Le premier thème comporte trois sous-thèmes : l'environnement socio-familial de l'enfant, les contraintes structurelles et les interactions avec le réseau de soins (tableau II). Le second se compose quant à lui des sous-thèmes suivants : la formation des professionnels de santé et la dimension relationnelle (tableau III).
Les thèmes 3 et 4 sont davantage en lien avec la psychiatrie adulte. Le thème 3 représente $47 \%$ du corpus et se rapporte à l'exercice dans le système hospitalier public avec trois sous-thèmes : le rôle du médecin-gestionnaire, la perte de sens du travail et la satisfaction au travail (tableau IV). Enfin, le dernier thème, correspondant à $12 \%$ du corpus, concerne la spécificité du travail en psychiatrie avec les aspects médico-légaux d'une part et la violence d'autre part (tableau V).

\section{Discussion}

Le vocabulaire employé et les extraits d'entretiens des 16 psychiatres ayant accepté de participer à l'étude ont permis de documenter une réflexion autour des quatre thèmes suivants.

\section{Prise en charge médico-sociale du patient}

Les pédopsychiatres déclarent être de plus en plus sollicités et confrontés à des difficultés en lien avec l'environnement sociofamilial de l'enfant (tableau II, réf. 1 et 2). En pédopsychiatrie, comme en psychiatrie adulte, les attentes de la société et des pouvoirs publics sont fortes à l'égard de cette spécialité qui doit à présent faire face à la « psychiatrisation » des problèmes sociaux, eux-mêmes accrus par

Tableau I : Thèmes et sous-thèmes issus de l'analyse du vocabulaire des entretiens

\begin{tabular}{|c|c|c|c|c|}
\hline & Thème 1 & Thème 2 & Thème 3 & Thème 4 \\
\hline 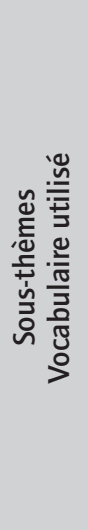 & $\begin{array}{l}\text { Environnement socio-familial } \\
\text { de l'enfant } \\
\text { Enfant, petit, confronter, } \\
\text { situation, parent, familial, } \\
\text { famille, recevoir, pression, } \\
\text { éducation, domicile } \\
\text { Contraintes structurelles } \\
\text { Secteur, lieu, structure, CMPa, } \\
\text { accueillir, accueil, place, délai, } \\
\text { confronter, suivre } \\
\text { Interactions avec le réseau de } \\
\text { soins } \\
\text { Secteur, CMP, hôpital, pédiatrie, } \\
\text { suivre, scolaire, solliciter, } \\
\text { intervenir, psycho, CATTPb }\end{array}$ & $\begin{array}{l}\text { Formation des professionnels } \\
\text { de santé } \\
\text { Qualité, soin, professionnel, } \\
\text { formation, personnel, penser, } \\
\text { connaissance, capacité, utile } \\
\text { Dimension relationnelle } \\
\text { Liaison, relation, mouvement, } \\
\text { participe, égard, équipe, regard, } \\
\text { bienveillant, personnel }\end{array}$ & $\begin{array}{l}\text { Rôle du médecin-gestionnaire } \\
\text { Management, position, chef, } \\
\text { prendre, décision, règle, } \\
\text { directeur, cadre } \\
\text { Perte de sens du travail } \\
\text { Administratif, impression, } \\
\text { individu, collectif, règle, parler, } \\
\text { valeur } \\
\text { Satisfaction au travail } \\
\text { Venir, boulot, envie, content, } \\
\text { bosser }\end{array}$ & $\begin{array}{l}\text { Aspects médico-légaux } \\
\text { Contrainte, certificat, signer, } \\
\text { traiter, hospitalisation, garde, } \\
\text { loi, marquer, mot, dossier, } \\
\text { pratique, juge, perdre, liberté, } \\
\text { couvrir, pratique, époque, } \\
\text { condamner } \\
\text { Violence } \\
\text { Danger, agressif, frapper, taper, } \\
\text { sédation, contenu, chambre, } \\
\text { isolement, peur }\end{array}$ \\
\hline
\end{tabular}

a : Centre Médico-Psychologique.

b : Centre d'Accueil Thérapeutique à Temps partiel. 
Tableau II : Illustration du thème 1 et des sous-thèmes par des extraits issus de l'analyse des entretiens

\begin{tabular}{|c|c|c|c|}
\hline \multirow[t]{3}{*}{ Thème 1} & \multicolumn{3}{|c|}{ Prise en charge médico-sociale du patient } \\
\hline & \multirow{2}{*}{$\begin{array}{l}\text { Environnement } \\
\text { socio-familial } \\
\text { de l'enfant }\end{array}$} & $\begin{array}{l}\text { "c'est beaucoup en pédopsy des demandes par rapport à la scolarité, par rapport aussi aux situations } \\
\text { familiales, c'est vrai que la famille vit un petit peu une crise (...) les parents divorcent, les familles se } \\
\text { recomposent, les enfants sont au milieu, et souvent il y a des complications" }\end{array}$ & $(1)$ \\
\hline & & $\begin{array}{l}\text { "on voit de plus en plus effectivement des familles en difficulté sociale et donc aussi tout ce qui concerne } \\
\text { le suivi des enfants placés" }\end{array}$ & $(2)$ \\
\hline \multirow{7}{*}{ 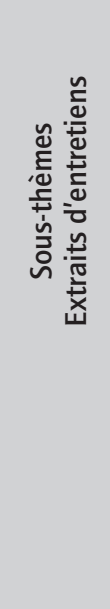 } & \multirow{5}{*}{$\begin{array}{l}\text { Contraintes } \\
\text { structurelles }\end{array}$} & "manque de places par rapport aux structures institutionnelles" & (3) \\
\hline & & "délais d'attente qui sont importants" & (4) \\
\hline & & "si ce n'est pas urgent, c'est de l'ordre de six mois un an d'attente" & $(5)$ \\
\hline & & "on ne dispose pas des locaux" & $(6)$ \\
\hline & & $\begin{array}{l}\text { "un manque de temps, un manque de moyens" } \\
\text { "cet aspect manque de temps, pression, effectivement je crois avoir le sentiment de ne pas arriver à } \\
\text { répondre à toutes les demandes (...) le sentiment d'être un petit peu dispersé, éclaté, de travailler dans } \\
\text { différents lieux sans forcément qu'il y ait de lien de continuité" }\end{array}$ & $\begin{array}{l}(7) \\
(8)\end{array}$ \\
\hline & & $\begin{array}{l}\text { "on envisage en CMPa à ce moment-là de combiner des soins de rééducation orthophonique ou } \\
\text { psychomotrice et un groupe thérapeutique hebdomadaire" }\end{array}$ & (9) \\
\hline & $\begin{array}{l}\text { Interactions avec } \\
\text { le réseau de soins }\end{array}$ & $\begin{array}{l}\text { "par le biais soit du médecin scolaire, soit de la psychologue scolaire, donc quand les enfants ont des } \\
\text { troubles du comportement importants (...) ou voilà, là il y a une pression de ce côté-là et puis aussi par le } \\
\text { Conseil général c'est-à-dire les Maisons du Rhône qui (...) reçoivent aussi des familles en difficulté et donc } \\
\text { nous sollicitent pour les suivre" }\end{array}$ & (10) \\
\hline
\end{tabular}

a : Centre Médico-Psychologique.

Tableau III : Illustration du thème 2 et des sous-thèmes par des extraits issus de l'analyse des entretiens

\begin{tabular}{|c|c|c|c|}
\hline Thème 2 & \multicolumn{3}{|c|}{ Qualité des soins } \\
\hline \multirow{3}{*}{ 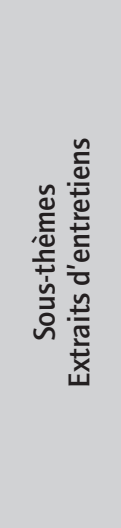 } & \multirow{2}{*}{$\begin{array}{l}\text { Formation } \\
\text { des } \\
\text { professionnels } \\
\text { de santé }\end{array}$} & $\begin{array}{l}\text { "on a besoin que les équipes soient formées, qu'elles soient formées de façon cohérente à des choses qui } \\
\text { sont adaptées à leur vrai besoin de formation" }\end{array}$ & (1) \\
\hline & & $\begin{array}{l}\text { "je pense que ça dépend en premier lieu de l'implication des soignants, tout à l'heure je disais que j'ai une } \\
\text { équipe qui n'est pas très bien formée, mais ils sont tellement impliqués que du coup (...) ça pallie un peu à } \\
\text { ce manque de formation" }\end{array}$ & (2) \\
\hline & $\begin{array}{l}\text { Dimension } \\
\text { relationnelle }\end{array}$ & $\begin{array}{l}\text { "la disponibilité du professionnel, de sa bienveillance, de ses compétences, de l'équipe qui l'entoure et du } \\
\text { fonctionnement institutionnel dans lequel il travaille et de la communication qui existe entre les différents } \\
\text { professionnels" } \\
\text { "la qualité des soins elle est aussi garantie par la qualité des liaisons avec les équipes partenaires, ça } \\
\text { c'est sûrement une vraie difficulté, on est dans des institutions qui ont des mandats différents et qui ne } \\
\text { sont pas faites pour communiquer ensemble" } \\
\text { "cette qualité de la relation n'est pas exclusivement la qualité de la relation soignante (...) elle ne peut } \\
\text { pas être isolée de la qualité de la relation soignante, de la qualité de la relation au sein d'une équipe, } \\
\text { et de la relation avec les collègues" }\end{array}$ & (4) \\
\hline
\end{tabular}

le contexte actuel de crise socio-économique. Cet élargissement des limites de la spécialité et la banalisation des soins psychiatriques augmentent ainsi considérablement la charge de travail [6]. La difficulté croissante, pour les pédopsychiatres interrogés, à répondre à toutes les demandes et la pression ainsi engendrée sont mises en évidence par les fréquentes co-occurrences des mots : famille, recevoir, pression.

Les contraintes d'ordre structurel sont soulignées par des mots tels que secteur, lieu, structure, CMP, accueillir, place, délai. Certains psychiatres exerçant en CMP rapportent un «manque de places par rapport aux structures institutionnelles», et de ce fait «des délais d'attente qui sont importants » (tableau II, réf. 3 et 4). D’autres dénoncent un manque de locaux (tableau II, réf. 6) ou bien des locaux vétustes ou inadaptés. Beaucoup évoquent également «un manque de temps, un manque de moyens » (tableau II, réf. 7). Or, la psychiatrie est une spécialité fondée sur la relation à l'autre où l'accompagnement du patient prend une place considérable dans le soin. Ce manque de temps conduit à un 
Tableau IV : Illustration du thème 3 et des sous-thèmes par des extraits issus de l'analyse des entretiens

\begin{tabular}{|c|c|c|c|}
\hline Thème 3 & \multicolumn{3}{|c|}{ Exercice dans le système hospitalier public } \\
\hline \multirow{3}{*}{ 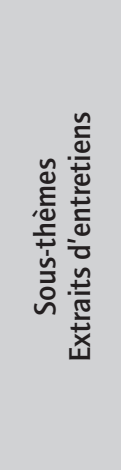 } & $\begin{array}{l}\text { Rôle du } \\
\text { médecin- } \\
\text { gestionnaire }\end{array}$ & $\begin{array}{l}\text { "(...) je fais beaucoup moins de clinique que de travail de responsabilité (...)" } \\
\text { "moi j'ai plus, là il me semble, évoqué des affaires de management, que l'on n'apprend pas, } \\
\text { qu'on ne nous apprend pas" }\end{array}$ & $\begin{array}{l}(1) \\
(2)\end{array}$ \\
\hline & $\begin{array}{c}\text { Perte du sens } \\
\text { du travail }\end{array}$ & $\begin{array}{l}\text { "progressivement, on en devient plus ou moins prestataire de services au lieu d'être, on va dire, un } \\
\text { élément moteur de la politique de soins " } \\
\text { "je ne me prive pas de le dire aux visiteurs de l'HAS a qui viennent nous certifier, je pense qu'à un } \\
\text { moment donné, à force de vouloir tout codifier, on a perdu un certain bon sens et on n'accepte plus } \\
\text { de prendre des risques et soigner c'est prendre un risque" } \\
\text { "le poids de l'administratif et des papiers à rendre et (...) des formulaires (...) aussi prend sur le temps } \\
\text { clinique. Donc c'est vraiment conjoncturel mais pénible, parce qu'avant tout on est médecin " }\end{array}$ & (4) \\
\hline & $\begin{array}{l}\text { Satisfaction } \\
\text { au travail }\end{array}$ & $\begin{array}{l}\text { "je suis satisfaite de venir travailler" } \\
\text { "je suis très contente de ce que je fais. C'est fatigant mais c'est bien " }\end{array}$ & $\begin{array}{l}(6) \\
(7)\end{array}$ \\
\hline
\end{tabular}

a : Haute Autorité de santé.

Tableau V : Illustration du thème 4 et des sous-thèmes par des extraits issus de l'analyse des entretiens

\begin{tabular}{|c|c|c|c|}
\hline Thème 4 & & Spécificité du travail en psychiatrie & \\
\hline \multirow{5}{*}{ 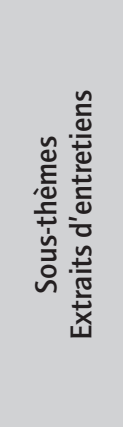 } & \multirow{3}{*}{$\begin{array}{l}\text { Aspects } \\
\text { médico-légaux }\end{array}$} & $\begin{array}{l}\text { "je n'ai pas d'avis favorable ou défavorable par rapport à la loi mais c'est sûr, ça a influencé } \\
\text { la pratique" }\end{array}$ & (1) \\
\hline & & $\begin{array}{l}\text { "passer devant le juge, déjà c'est extrêmement compliqué, souvent ils ne comprennent pas, alors on } \\
\text { explique bien que c'est sur (...) la forme (...) plus que sur le fond, mais déjà ça, ce n'est pas évident " }\end{array}$ & $(2)$ \\
\hline & & $\begin{array}{l}\text { "le certificat médical initial de SDRE a n'a pas été suffisamment étayé, bien que l'hospitalisation était } \\
\text { tout à fait justifiée et que la mise sous traitement était tout à fait justifiée, mais il a été condamné } \\
\text { un truc symbolique" }\end{array}$ & (3) \\
\hline & \multirow[b]{2}{*}{ Violence } & "c'est une violence faite au patient que de le contenir (...)" & (4) \\
\hline & & $\begin{array}{l}\text { "j'ai eu à subir des agressions où j'ai toujours trouvé un moyen de gérer la situation de crise, } \\
\text { sauf une fois quand j'étais interne" }\end{array}$ & (5) \\
\hline
\end{tabular}

a : Soins psychiatriques sur décision du représentant de l'État.

sentiment de disponibilité moindre et de pression supplémentaire (tableau II, réf. 8). Selon l'enquête santé et satisfaction des médecins au travail (SESMAT), le manque de temps pour parler au patient apparaît relié à l'épuisement professionnel des psychiatres [5]. Des études concernant l'hôpital psychiatrique montrent que les moyens attribués au système de soins s'amenuisent (réduction du nombre de lits, du nombre de soignants, etc.) tandis que paradoxalement la charge de travail s'alourdit $[6,17,18]$.

Le développement de l'extra-hospitalier induit pour les médecins une activité multi-sites plus fréquente avec une multiplication de partenaires médicaux, paramédicaux et sociaux [6]. Ces interactions avec le réseau de soins sont caractérisées par des mots comme secteur, CMP, hôpital, pédiatrie, suivre, scolaire, solliciter, intervenir (tableau II, réf. 9 et 10). Ce travail multi-sites est à l'origine d'une réduction des pauses-repas et multiplie par deux la survenue de l'épuisement professionnel [5].

\section{Qualité des soins}

Selon l'Organisation Mondiale de la Santé, la qualité des soins se définit comme la capacité de « garantir à chaque patient l'assortiment d'actes diagnostiques et thérapeutiques, qui lui assure le meilleur résultat en termes de santé, conformément à l'état de la science médicale, au meilleur coût pour un même résultat, au moindre risque iatrogène et pour la plus grande satisfaction en termes de procédures, de résultats et de contacts humains à l'intérieur du système de soins » [24]. Cependant, des études réalisées auprès du personnel hospitalier sur la signification et la mesure de la qualité des soins soulignent l'absence de définition unique et la différence de représentation entre encadrants et soignants $[25,26]$. Pour ces derniers, la qualité des soins est centrée sur les aspects relationnels et inscrite dans une pratique collective professionnelle. 
Les mots qualité, soin, professionnel, formation, personnel, connaissance, utile se réfèrent à la formation des professionnels de santé. Certains psychiatres évoquent le manque de formation de l'équipe infirmière (tableau III, réf. 1). L'enquête PRESST-NEXT fait état également de ce ressenti d'une déficience de formation du personnel paramédical en psychiatrie, en lien notamment avec la disparition depuis 1992 du diplôme infirmier de secteur psychiatrique. Ce ressenti est exprimé aussi bien par les infirmiers que par la hiérarchie [17]. Cependant, un des psychiatres interrogés déclare que ce manque de formation peut être compensé dans certains cas par l'investissement du soignant (tableau III, réf. 2). Afin d'améliorer la formation infirmière, une nouvelle formation initiale ( $1^{\text {re }}$ promotion 2009-2012) doit être évaluée en tenant compte de son impact sur la qualité des soins [27].

Tous les psychiatres évoquent la dimension relationnelle en employant des mots tels que liaison, relation, mouvement, participe, égard, équipe, regard (tableau III, réf. 3 et 4). L'importance de la relation soignant-soigné, avec les places primordiales de l'écoute et de l'échange, est rapportée dans toutes les études concernant les conditions de travail en psychiatrie $[5,6,17,18]$. Les soignants déplorent la rationalisation du temps au détriment du relationnel. Leur perception globale est celle d'une détérioration de la qualité des soins et de la relation soignant-soigné $[18,28]$. Certains dénoncent l'évolution de la conception du soin de «la relation humaine soignant/soigné » à une conception «efficacité » [29]. En outre, comme le signale l'un des psychiatres de l'étude, la relation soignant-soigné ne peut être dissociée de la relation soignant-soignant (tableau III, réf. 5). L'enquête SESMAT montre que la survenue de l'épuisement professionnel est doublée en cas de relations interpersonnelles hostiles ou tendues dans l'équipe et quasiment multipliée par trois lorsque la qualité du travail d'équipe est déclarée faible [5]. Les possibilités d'échange dans l'équipe apparaissent déterminantes, notamment sous leur modalité informelle. Les psychiatres de notre étude déplorent ainsi la disparition progressive de ces temps informels. Ceux-ci aident en effet à diluer une part du stress chronique en permettant les décharges émotionnelles autant que les réassurances en cas de difficultés rencontrées [5].

\section{Exercice dans le système hospitalier public}

Les trois chefs de pôle interrogés évoquent leur nouvelle fonction de responsable de gestion (tableau IV, réf. 1) instituée lors de la création des pôles d'activités par la réforme de la Nouvelle gouvernance hospitalière puis le Plan Hôpital en 2007. Dans notre étude, des mots tels que management, position, chef, prendre, décision, directeur, cadre se rapportent au rôle du médecin-gestionnaire. Cette fonction dépasse à présent le seul champ de la médecine du fait de l'introduction d'outils gestionnaires et du phénomène de «managérialisation» à l'hôpital public [30]. L'émergence d'un nouvel équilibre entre pouvoir médical et pouvoir gestionnaire [31] renforce les mécanismes de domination entre médecins et gestionnaires [32]. Ceci s'ajoute aux contraintes rencontrées par l'activité psychiatrique depuis la loi de 1990, en particulier la difficulté de délimiter les tâches des institutions et des professionnels ainsi que la relation entre l'offre de soins des établissements et la permanence des soins.

La rationalité économique et la culture du résultat envahissent l'activité de soins et prennent le pas sur les relations humaines [18]. Les réformes instaurées cette dernière décennie sont associées pour beaucoup de soignants à une certaine perte de sens du travail (tableau IV, réf. 3), illustrée dans leurs propos par les mots administratif, impression, individu, collectif, règle, parler, valeur. Les nouvelles démarches de qualité et de certification représentent une vision des soins qualifiée de procédurale par les soignants en psychiatrie (tableau IV, réf. 4). Ce système n'évalue pas le processus réel du travail et oublie la subjectivité et la compréhension de l'autre [33]. Cette représentation est en contradiction avec leur propre conception, centrée sur le temps, la disponibilité et le relationnel [18]. Ces procédures introduisent une prescription du travail de plus en plus individualisée. Elle va ainsi à l'encontre de la culture du personnel soignant car perçue comme aboutissant à la dislocation du collectif de travail et à la remise en cause des valeurs associées à l'identité professionnelle [34]. La part de temps consacrée à l'activité administrative augmente (tableau IV, réf. 5) au détriment de l'écoute du patient, contribuant à l'augmentation des situations de violence, qui sont plus difficilement anticipées. Cette organisation crée un décalage entre «prescrit et réel » formant un environnement porteur de violence [35]. Aussi, bien que les changements organisationnels n'affectent pas spécifiquement le secteur psychiatrique, ils ont cependant un retentissement plus fort dans des situations déjà porteuses de violence [18]. La perte de sens du travail constitue un facteur de risque de l'épuisement professionnel [18]. Dans ce cadre, de nombreuses résistances à la culture du résultat apparaissent en psychiatrie [36].

Historiquement, des glissements de tâches s'opèrent fréquemment en service de psychiatrie. Des études récentes ont mis en évidence une réduction des possibilités de 
coopération notamment entre catégories professionnelles au sein des équipes de psychiatrie par l'introduction de la division formalisée du travail [37]. Les soignants n'ont plus la possibilité de construire les « savoir-faire de prudence» essentiels dans la prévention des situations de crise [18].

Néanmoins la plupart des psychiatres interrogés sont satisfaits de leurs activités professionnelles, comme l'attestent les mots venir, boulot, envie, content, bosser (tableau IV, réf. 6 et 7). La satisfaction professionnelle serait un facteur protecteur de l'épuisement professionnel $[8,38]$. La conservation d'un état de satisfaction au travail est souvent rapportée dans les études concernant les psychiatres hospitaliers malgré un niveau d'épuisement professionnel parfois avancé [6, 39].

\section{Spécificité du travail en psychiatrie}

Dans cette étude, l'influence des nouvelles réglementations sur la pratique quotidienne est évoquée par les psychiatres (tableau $\mathrm{V}$, réf. 1 et 2 ). Les aspects médicolégaux sont caractérisés par les mots mettre, mot, marquer, dossier, traiter, loi, pratique, lever, contrainte, juge, perdre, liberté, signer. Une étude réalisée six mois après l'application de la loi du 5 juillet 2011, relative aux droits et à la protection des personnes faisant l'objet de soins psychiatriques et aux modalités de leur prise en charge, montre que cette dernière apporte certains avantages mais aussi des difficultés : articulation entre univers médical et judiciaire complexe, manque de moyens, certificats médicaux trop nombreux, etc. [40, 41]. Les mots couvrir, époque, condamner, certificat sont utilisés pour évoquer certaines situations ayant été vécues par des confrères (tableau V, réf. 3). L'enquête PRESST-NEXT indique que les nouvelles directives législatives et réglementaires afférentes aux droits des malades et aux obligations des structures de soins laissent place à la judiciarisation et aux conflits avec les usagers, amplifiant ainsi le risque médico-légal [17].

La violence est caractérisée par des mots tels que danger, agressif, frapper, taper, sédation, contenu, chambre, isolement, peur. Sont citées à la fois les situations violentes que peuvent vivre certains patients (tableau $V$, réf. 4 ) et celles vécues par les soignants (tableau $V$, réf. 5). En psychiatrie, le risque d'exposition à des situations de violence est élevé. Cependant, il existe une certaine forme de tolérance de la part des praticiens. En effet, ils attribuent cette violence à la maladie mentale et non directement au patient. Cette tolérance constitue un élément non négligeable du stress vécu au travail [42].

\section{Limites de l'étude}

L'interprétation des résultats de cette enquête doit rester prudente, la faible taille de l'échantillon ne le rendant pas représentatif de l'ensemble des psychiatres hospitaliers. Le choix d'entretiens semi-directifs réalisés par un seul enquêteur peut comporter un biais lié à la directivité. Toutefois, la part de subjectivité dans l'interprétation des entretiens a pu être réduite par l'utilisation du logiciel Alceste. Comme pour toute étude qualitative, ce n'est pas la représentativité de la population qui a été souhaitée mais celle de la diversité des situations (âge, sexe, secteur) [19,20]. Celle-ci a été augmentée en réalisant les entretiens sur deux établissements différents. Des facteurs de confusion comme le fait de travailler exclusivement en secteur ouvert ou fermé ou bien en CMP ou en milieu carcéral n'ont pas été choisis car la majorité des psychiatres de ces établissements travaillaient dans plusieurs services à la fois. De même, la spécialité en addictologie n'a pas été retenue. Ces caractéristiques sont néanmoins à étudier et pourront faire l'objet d'une enquête ultérieure.

\section{Conclusions}

Il apparaît au travers des entretiens des psychiatres que parmi les éléments à améliorer en priorité figurent une organisation du temps de travail favorisant l'écoute des patients, les échanges interprofessionnels, notamment au cours de moments informels ainsi que la formation du personnel paramédical en psychiatrie. Ce travail a permis d'élaborer un questionnaire spécifique qui pourra être appliqué à une plus large population de psychiatres hospitaliers.

Aucun conflit d'intérêt déclaré

\footnotetext{
Remerciements

Nous tenons à remercier tous les psychiatres qui ont participé à l'étude. Nous remercions pour leurs conseils le Professeur Naudin et les Docteurs Samuelian et Zendjidjian ainsi que Madame Chianese, psychologue.
} 


\section{Références}

1. Dupuis G, Martel JP, Voirol C, Bibeau L, Hébert-Bonneville N. La qualité de vie au travail : bilan des connaissances [Internet]. CLIPP ; 2009. Available from: <http://www.clipp.ca>.

2. Gollac M, Bodier M, eds. Mesurer les facteurs psychosociaux de risque au travail pour les maîtriser. Rapport du Collège d'expertise sur le suivi des risques psychosociaux au travail, faisant suite à la demande du Ministre du travail, de l'emploi et de la santé. Paris: Collège d'expertise sur le suivi des risques psychosociaux au travail ; 2011.

3. Haute Autorité de santé (HAS). Actes du séminaire "Qualité de vie au travail et qualité des soins dans les établissements de santé ". La Plaine Saint-Denis : HAS ; 21 octobre 2010.

4. Donato J. Une perception dégradée des conditions de travail. Santé \& Travail. 2009;(66).

5. Estryn-Behar M, BraudoMH, Fry C, Guetarni K. Enquête comparative sur le syndrome d'épuisement professionnel chez les psychiatres et les autres spécialistes des hôpitaux publics en France (enquête SESMAT). L'information psychiatrique 2011:87:95-117.

6. Wiertz R. Psychiatres et burnout? : Étude de la prévalence du syndrome d'épuisement professionnel auprès des psychiatres hospitaliers du Nord Pas De Calais. Thèse de Doctorat en Médecine, Université du droit et de la santé Lille 2, 2012. 224p.

7. Kumar S. Burnout and psychiatrists: what do we know and where to from here? Review. Epidemiol Psychiatr Sc. 2011;20(4):295-301.

8. Bressi C, Porcellana M, Gambini O, Madia L, Muffatti R, Peirone A, et al. Burnout among psychiatrists in Milan: a multicenter survey. Psychiatr Serv. 2009;60(7):985-8.

9. Umene-Nakano W, Kato TA, Kikuchi S, Tateno M, Fujisawa D, Hoshuyama T, et al. Nationwide Survey of Work Environment, WorkLife Balance and Burnout among Psychiatrists in Japan. PLoS One. 2013;8(2):1-8.

10. Korkeila JA, Töyry S, Kumpulainen K, Toivola JM, Räsänen K, Kalimo R. Burnout and self-perceived health among Finnish psychiatrists and child psychiatrists: a national survey. Scand J Public Health. 2003;31:85-91.

11. Hawton K, Clements A, Sakarovitch C, Simkin S, Deeks JJ. Suicide in doctors: a study of risk according to gender, seniority and specialty in medical practitioners in England and Wales, 1979-1995. J Epidemiol Community Health. 2001;55:296-300.

12. Rich $C L$, Pitts FN Jr. Suicide by psychiatrists: a study of medical specialists among 18,370 consecutive physician deaths during a five-year period, 1967-1972. J Clin Psychiatry. 1980;41(8):261-3.

13. Chocard AS, Juan F. Suicide et tentatives de suicide chez les médecins. La lettre du Psychiatre. 2007;3(1-2):10-3.

14. Fothergill $A$, Edwards D, Burnard P. Stress, burnout, coping and stress management in psychiatrists: findings from a systematic review. Int J Soc Psychiatry. 2004;50(1):54-65.

15. Kumar S. Burnout in psychiatrists. World Psychiatry. 2007;6(3): 186-9.

16. Rössler W. Stress, burnout, and job dissatisfaction in mental health workers. Eur Arch Psychiatry Clin Neurosci. 2012;262(Suppl 2):565-9.

17. Estryn-Behar M, Duville N, MeniniNL, Le Foll S, Le Nezet O, Bocher R. Mots à maux... Expression de la souffrance des soignants en psychiatrie. Étude comparative en France et dans 3 autres pays européens. Annales Médico Psychologiques. 2006;164:732-48.
18. Cintas C. Pénibilité du travail en hôpital psychiatrique. Pistes. 2009;11(1):1-17.

19. Britten N. Qualitative interviews in medical research.BMJ. 1995;311(6999):251-3.

20. Brod M, Tesler LE, Christensen TL. Qualitative research and content validity: developing best practices based on science and experience. Qual Life Res. 2009;18(9):1263-78.

21. Leplège A, Coste J. Mesure de la santé perceptuelle et de la qualité de vie- méthodes et applications. Paris : Éditions ESTEM ; 2001.

22. Blanchet A, Gotman A. L'enquête et ses méthodes - L'entretien. $2^{\mathrm{e}}$ éd. Paris : Éditions Armand Colin, Collection 128; 2007.

23. Reinert M. Les mondes lexicaux et leur logique à travers l'analyse statistique d'un corpus de récits de cauchemars. Langage et société. 1993;66(1):5-39.

24. Roemer MI, Montoya-Aguilar C, World health organization. Quality assessment and assurance in primary health care. Geneva: WHO Offset Publication, Report No.105; 1988.

25. Or Z, Com-Ruelle L. La qualité des soins en France : comment la mesurer pour l'améliorer? Paris : IRDES, DT n $19 ; 2008$.

26. Claveranne JP, Vinot D, Fraisse $S$, Robelet $M$, Candel D, Dubois D, et al. Les perceptions de la qualité chez les professionnels des établissements de santé. Paris: Centre national de la recherche scientifique, Rapport de recherche GRAPHOS-CNRS, LCPE/LNSTCNRS; 2003.

27. Ministère chargé de la santé, Ministère chargé des solidarités. Plan psychiatrie et santé mentale 2011-2015. Paris: les ministères. Available from: <www.sante.gouv.fr>.

28. Douguet F, Munoz J, Leboul D. Les effets de l'accréditation et des mesures d'amélioration sur la qualité des soins sur l'activité des personnels soignants. Paris: Direction de la recherche, des études, de l'évaluation et des statistiques, Série Études $n^{\circ} 48$; 2005.

29. Brient P. Les effets de la formation sur site auprès des soignants d'unités en psychiatrie générale : bilan d'une expérience. Pratiques Psychologiques. 2007;13(4):459-64.

30. Burellier F. Être ou ne pas être médecin-gestionnaire ? Étude de la transition vers le rôle de responsable de pôle au sein des hôpitaux publics français. Thèse de Doctorat de I'Université de Grenoble, spécialité sciences de gestion, Centre d'études et de recherches appliquées à la gestion (CERAG), École doctorale de sciences de gestion de Grenoble ; 2011. 421p.

31. Vallet-Armelllino M. Tomber juste. Des effets sur la clinique des nouvelles normes de gestion de la psychiatrie. Nouvelle revue de psychosociologie (Édition ERES). 2007;(4):143-59.

32. Sainsaulieu I. La communauté des soins en question. Paris : Éditions Lamarre; 2006.

33. Institut de psycho dynamique du travail au Québec. Espace de réflexion et espace d'action : enquêtes en psycho dynamique du travail au Québec. Québec: Presses Universitaires de Laval ; 2006.

34. Collectif infirmier (2007). À l'écoute de la folie avec un collectif d'infirmiers en psychiatrie. Nouvelle revue de psychosociologie. 2007;(4):159-81.

35. Dejours C. Travaux préparatoires du Plan violence et santé. Paris : Commission Violence, travail, emploi, santé, Conseil économique et social ; 2005.

36. Borgès Da Silva G. La qualité des soins en hôpital psychiatrique : revue de littérature et perspectives. Santé Publique. 2003;15(2): 213-22. 
37. Douguet F, Munoz J. Les effets de l'accréditation et des mesures d'amélioration sur la qualité des soins sur l'activité des personnels soignants. Paris: Direction de la recherche, des études, de l'évaluation et des statistiques, rapport $n^{\circ} 48 ; 2005$.

38. Fischer J, Kumar S, Hatcher S. What makes psychiatry such a stressful profession? A qualitative study. Australas Psychiatry. 2007; 15(5):417-21.

39. Kumar S, Fischer J, Robinson E, Hatcher S, Bhagat RN. Burnout and job satisfaction in New Zealand psychiatrists: a national study. Int J Soc Psychiatry. 2007;53(4):306-16.

40. République Française. Loi $n^{\circ} 2011-803$ du 5 juillet 2011 relative aux droits et à la protection des personnes faisant l'objet de soins psychiatriques et aux modalités de leur prise en charge. JORF. 6 juillet 2011;(0155):11705, texte $n^{\circ} 1$.

41. Cambier G, Bougerol T, Micheletti P. Enquête qualitative sur la loi du 5 juillet 2011 en psychiatrie. Santé Publique. 2013;25(6): 793-802.

42. Deleplancque N, Fabian M, QuievreuxJL, Masurel I, Vandamme M, Caby MJ, et al. Stress, Violence externe et interprofessionnelle: Étude de risques psychosociaux au sein de quinze fonctions proches du soin en psychiatrie. Étude multicentrique dans 3 EPSM du Nord de la France (Armentières Bailleul Saint André) [Internet]. 2009. Available from: <http://www.santementale 5962.com>.

\section{Annexe : Guide d'entretien}

- Que pouvez-vous me dire sur vos conditions de travail actuelles?

- Quand vous êtes au travail, que ressentez-vous?

- Est-ce que vous rencontrez des difficultés dans votre exercice?

- Quel est l'impact de l'organisation de travail sur votre façon de travailler?

- Dans votre activité, quelles sont vos responsabilités?

- Selon vous, de quoi dépend la qualité des soins?

- Que pouvez-vous me dire sur vos relations au travail en général?

- Avec votre hiérarchie

- Avec les médecins

- Avec les administratifs

- Avec vos pairs

- Avec les paramédicaux

- Que pouvez-vous me dire sur vos relations avec les patients et leur entourage?

- Que pensez-vous du lien entre votre vie professionnelle et votre vie personnelle?

- Comment évaluez-vous l'influence de votre travail sur votre santé?

- Que préconiseriez-vous pour améliorer votre qualité de vie au travail ? Et pour améliorer la qualité de vie au travail des autres?

- Souhaitez-vous ajouter quelque chose? 